\title{
Effect of mannose modified chitosan on uptake of nanoparticles by macrophages
}

\author{
David Lowsberg, and John Smith\# \\ Department of Biochemistry, University of South Carolina, Columbia SC, 29208 \\ \#Address: 631 Sumter Street, Columbia, SC 29208; Email: jsmith@medicine.usc.edu
}

\begin{abstract}
This report provided a new method to prepare and evaluate mannose-modified chitosan-coated lactic acid-glycolic acid copolymer (PLGA) nanoparticles, and to investigate their effects on macrophage toxicity and macrophage uptake. The PLGA nanoparticles loaded with ovalbumin (OVA) were prepared by double emulsion method. The size and zeta potential of the nanoparticles were determined by laser granulometry after mannose-modified chitosan coating. The nanoparticles were observed by transmission electron microscopy. The appearance of the form, the BCA method to determine the OVA content, calculate the drug loading and release. The OVA nanoparticles labeled with fluorescein isothiocyanate (FITC) were co-incubated with macrophages (RAW 264. 7), cell viability was determined by MTT assay, and uptake was examined by fluorescence microscopy. Results The size and $\zeta$ potential of OVAPLGA nanoparticles increased with the increase of chitosan coating concentration $(P<$ 0.05 ), and OVA drug loading range was $7.2 \%$ to $8.4 \%$. Chitosan and mannose modified chitosan coating FITC-OVAPLGA nanoparticles and RAW 264. 7 After incubation, there was little effect on cell viability $(P>0.05)$, but it significantly promoted macrophage uptake by FITC-OVA-PLGA nanoparticles $(P<0.05)$.
\end{abstract}

\section{Introduction}

Macrophages and dendritic cells are specialized antigen-presenting cells (APCs) in the body and play an important role in the prevention and treatment of natural and acquired immunity, inflammation and tumors [1-3]. By increasing the targeting and accessibility of vaccines to APCs ${ }^{1-6}$, the goal of promoting immune response and reducing side effects can be achieved $[4,5]$. Macrophages have the property of phagocytizing foreign particulate matter, and if a passive targeting strategy such as receptor-mediated nanocarrier administration is employed, the targeted delivery of antigen can be further improved $^{7-20}$. It has been reported that a large number of C-type lectin family transmembrane protein-mannose receptors are expressed on the surface of macrophages to recognize polysaccharide molecules and soluble glycoproteins on the surface of cells or pathogens, and through ligand-receptor binding, Endocytosis or 
phagocytosis eliminates pathogens and excess glycoproteins in the body [6]. Yeeprae et al [7] proposed the effect of ligand density on cell recognition and phagocytosis by preparing mannose-modified OM emulsion. Zhou et al [8] applied mannose modified chitosan microspheres to improve the immune response of hepatitis $B$ virus DNA, indicating that mannose modification improved the release of chitosan microspheres and membrane permeability. In this study, ovalbumin (OVA) was used as a model antigen to prepare biodegradable polylactic-coglycolic acid (PLGA) nanoparticles by double emulsion method, and chitosan was modified by self-made mannose. After the coating treatment, the effect on the uptake function of macrophages was investigated to provide a basis for the research of new vaccine preparations.

\section{Materials and Method}

Reagent fluorescein isothiocyanate (FITC) -OVA and mannose modified chitosan (ManCS) made by our laboratory; lactic acid-glycolic acid copolymer (PLGA, 50: 50, molecular weight 20 000) was purchased from Jinan Biotech Co., Ltd.; Chitosan (CS, molecular weight $8000 \sim 10$ 000) purchased from Nantong Xingcheng Biological Products Factory; 2,2'-biquinoline-4, 4'-dicarboxylic acid disodium ( BCA, purity: BR) purchased from Suzhou Industrial Park Yake Chemical Reagent Co., Ltd.; DMEM high sugar medium purchased from Gibco; Newborn bovine serum purchased from Hangzhou Sijiqing Biotechnology Co., Ltd.; Trypsin, glutamine (purity: BR) purchased from Sinopharm Chemical Reagent Co., Ltd.; Hepes and DAPI staining solution were purchased from Biyuntian Biotechnology Research Institute, Haimen; Macrophage (RAW 264. 7) was purchased from Shanghai Library of Chinese Academy of Sciences. Preparation of Man-CS coated PLGA nanoparticles Preparation of OVA-PLGA nanoparticles Reference [9]: Add $250 \mu \mathrm{l}$ of $10 \mathrm{mg}$ OVA in polyvinyl alcohol (PVA) solution (mass concentration 3\%) to PLGA acetic acid In the ethyl ester solution, the colostrum (W1 /O) was prepared by ultrasonic bathing for $10 \mathrm{~s}$ (200 W, working $1 \mathrm{~s} /$ interval $1 \mathrm{~s}$ ). Then add colostrum to the PVA solution (mass concentration $3 \%$ ), stir evenly in the ice bath (15 $000 \mathrm{r} / \mathrm{min} \times 5 \mathrm{~min})$, phacoemulsification (400W, work $1 \mathrm{~s} /$ interval $1 \mathrm{~s}$ ), forming oil-in-water Water-containing double emulsion (W1 / O / W2). Finally, the double emulsion was transferred to a PVA solution $(20 \mathrm{ml}$, mass concentration of $0.5 \%$, and magnetically stirred to a completely volatile organic solvent at room temperature, centrifuged (3 $000 \mathrm{r} / \mathrm{min} \times 10 \mathrm{~min}$ ), and the precipitate was discarded. The supernatant was centrifuged (19 $000 \mathrm{r} / \mathrm{min} \times 20 \mathrm{~min})$, and the precipitate was collected and washed 3 times with deionized water. Pre-frozen $\left(-70{ }^{\circ} \mathrm{C}\right)$, freezedried, that is, PLGA nanoparticles. CS or Man-CS coating: The above double emulsion is directly added to a PVA acetic acid solution containing a certain concentration of CS or Man-CS (mass concentration of $0.5 \%$ ), the organic solvent is volatilized at room temperature, centrifuged, washed 3 times $^{21-27}$, frozen Dry is available. Nanoparticle preparation evaluation. Nanoparticle size and $\zeta$ Potential Determination The nanoparticle colloidal solution or lyophilized nanoparticles dispersed in distilled water 
were taken, and the size of the nanoparticles and the zeta potential were measured by a laser particle size analyzer. Nanoparticle morphology observation The nanoparticle solution was diluted to the appropriate concentration, stained with $2 \%$ phosphotungstic acid, dropped onto a copper mesh, and observed under a transmission electron microscope. Encapsulation rate and drug loading determination Precision weighed lyophilized nanoparticles $20 \mathrm{mg}$, added to $1 \mathrm{ml} 5 \%$ sodium lauryl sulfate / 0 . In a $1 \mathrm{~mol}$ / $\mathrm{L} \mathrm{NaOH}$ solution, shake at a constant temperature of $25^{\circ} \mathrm{C}$ for $4 \mathrm{~h}$, centrifuge $(10000 \mathrm{r}$ / $\mathrm{min}$ ) for $10 \mathrm{~min}$. The OVA content in the supernatant was determined by the BCA method, and the loading amount (LA) and the entrapment efficiency (EE) were calculated according to the following formula: $L A=$ the amount of protein in the nanoparticles. The mass of the nanoparticles $\times 100 \% \mathrm{EE}=$ amount of protein in the nanoparticles. Total amount of protein added $\times 100 \%$. Nanoparticle release in vitro Precision weighed lyophilized nanoparticles $50 \mathrm{mg}$ Dispered in 1. In a $5 \mathrm{ml}$ EP tube, add $1 \mathrm{ml}$ of poloxamer 188 solution (mass concentration $0.02 \%, \mathrm{PBS}, \mathrm{pH}=7.4$ ), shake at $37^{\circ} \mathrm{C}$, and accurately remove at a predetermined time. $5 \mathrm{ml}$, centrifuge $(19000 \mathrm{r} / \mathrm{min})$ for $15 \mathrm{~min}$. The supernatant was taken, the protein concentration was determined by the BCA method, the release was calculated, and the same volume of the release medium was added. Man-CS-OVA-PLGA Nanoparticle Cytotoxicity Test Rapidly thaw frozen macrophages (RAW 264. 7) at $37{ }^{\circ} \mathrm{C}$, add $7 \mathrm{ml}$ of DMEM high glucose medium, centrifuge twice at $1000 \mathrm{r} / \mathrm{min}$. Add DMEM high glucose medium containing $10 \%$ calf serum (containing $100 \mathrm{U} / \mathrm{ml}$ penicillin and streptomycin) in $37^{\circ} \mathrm{C}, 5 \% \mathrm{CO} 2$ incubator, change the culture medium on the 2 nd day, the cells are covered $70 \% \sim 80$ When $\%$, use $0.25 \%$ trypsin digestion. The cells were seeded in 96 -well plates at $5 \times 103 /$ well $^{28-}$ ${ }^{32}$, and cultured until the cells were attached. The experimental group was added with free FITC-OVA, PLGA nanoparticles containing FITC-OVA, and chitosan-coated PLGA (CS-PLGA). Nanoparticles and mannose-modified CS-coated PLGA (Man-CS-PLGA) nanoparticles. Another cell blank control well is set, and 6 replicate wells are provided for each well. After culturing for a certain period of time in the incubator, 96-well plates were taken, $10 \mu \mathrm{l}$ of medium was aspirated per well, and $10 \mu \mathrm{l}$ of tetramethyl azozolium salt (MTT) was added, and incubation was continued for $4 \mathrm{~h}$. All the supernatant was aspirated, and $100 \mu \mathrm{l}$ of SDS- $\mathrm{HCl}$ was added to each well overnight. The absorbance (A) was measured at $570 \mathrm{~nm}$ with a microplate reader, and the cell viability was calculated by adjusting the blank of the medium to zero. 5 Macrophage uptake experiments. The trypsin-digested logarithmic growth macrophages were seeded in 6-well plates $(5 \times 105$ $/ \mathrm{ml}$ ) at $2 \mathrm{ml}$ per well and cultured overnight. Then, PBS, free FITC-OVA, FITC-OVAPLGA nanoparticles, CS-PLGA nanoparticles and Man-CS-PLGA nanoparticles were added and incubated for $8 \mathrm{~h}$. The supernatant was discarded and washed 3 times with PBS and observed under a fluorescent microscope. In the above test, the concentration of FITC-OVA was $20 \mu \mathrm{g} / \mathrm{ml}$. Statistical processing. The measurement data is expressed as $\mathrm{x} \pm \mathrm{s}$, using SPSS 16. 0 Statistical software for one-way ANOVA, $\mathrm{P}<0$. 05 indicates that the difference is statistically significant. 


\section{Results and discussion}

The effect of CS or Man-CS coating on PLGA nanoparticle size, $\zeta$ potential and drug loading is shown in Table 1. It can be seen from Table 1 that the chitosan coating increases the particle size of PLGA nanoparticles and tends to increase with the increase of chitosan concentration $(P<0.05)$. The zeta potential of the PLGA nanoparticles was negative and was converted to a positive value after chitosan coating and was affected by the chitosan concentration $(P<0.05)$. However, the chitosan coating concentration had little effect on the drug loading of OVA $(P>0.05)$.
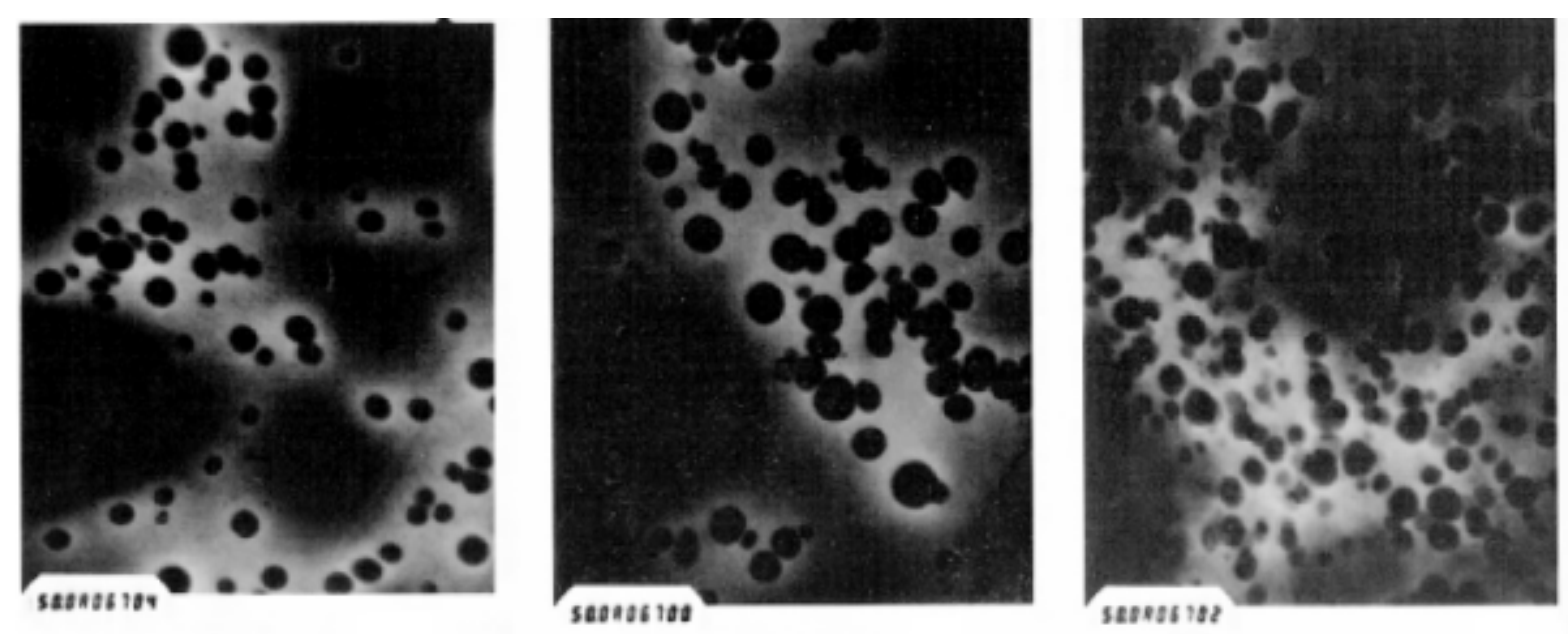

Figure 1. TEM analysis of nanoparticles.

The release of OVA-PLGA nanoparticles showed significant burst and sustained release characteristics. The $1 \mathrm{~h}$ release rate of OVA exceeded $30 \%$, followed by release near zero-order velocity, and the release rate reached $80 \%$ at $30 \mathrm{~d}$. OVA-PLGA via CS After coating with Man-CS, OVA burst release was reduced, but the percentage of total OVA release was also significantly reduced (both $P<0.05$ ) (Figure 2 ).

Macrophages were incubated with free FITC-OVA, FITCOVA-PLGA nanoparticles, CScoated PLGA (CS-PLGA) nanoparticles, and mannose-modified chitosan-coated PLGA (Man-CS-PLGA) nanoparticles. At $8 \mathrm{~h}$, the viability of the cells measured by the MTT method is shown in Figure 3. As can be seen from Figure 3, free FITC-OVA, FITC-OVAPLGA nanoparticles, CS-PLGA nanoparticles, and Man-CS coated PLGA nanoparticles at a concentration of 10 to $80 \mu \mathrm{g} / \mathrm{ml}$ were co-incubated with macrophages. Except for Man-CS-PLGA nanoparticles with $80 \mu \mathrm{g} / \mathrm{ml}$ concentration, cell viability was higher than $90 \%$. Analysis of variance showed no significant difference $(P>0.05)$. 


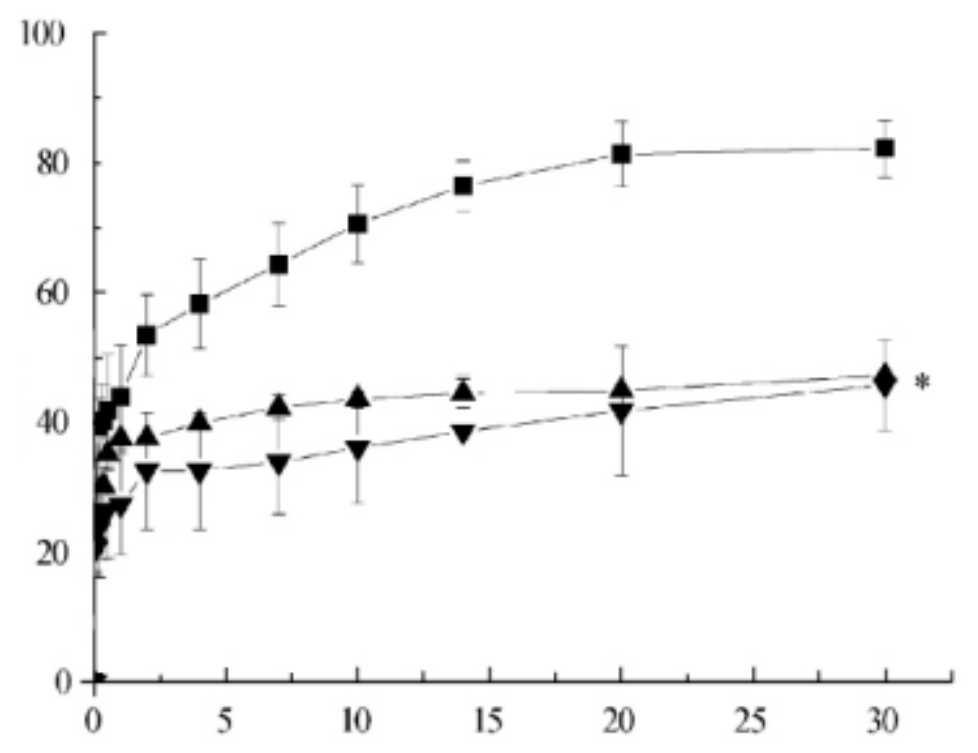

Figure 2. Releasing kinetics of nanoparticles.

The effect of Man-CS coating on the uptake function of macrophage FITC-OVA-PLGA nanoparticles is shown in Figure 4. As can be seen from Figure 4, the fluorescence intensity of macrophages was weak after co-incubation with free FITC-OVA solution. However, the fluorescence intensity of macrophages was significantly increased after incubation with FITC-OVA-PLGA nanoparticles. The macrophages co-incubated with Man-CS coated FITC-OVA-PLGA nanoparticles showed the strongest fluorescence, followed by CS coated FITC-OVA-PLGA nanoparticles. 


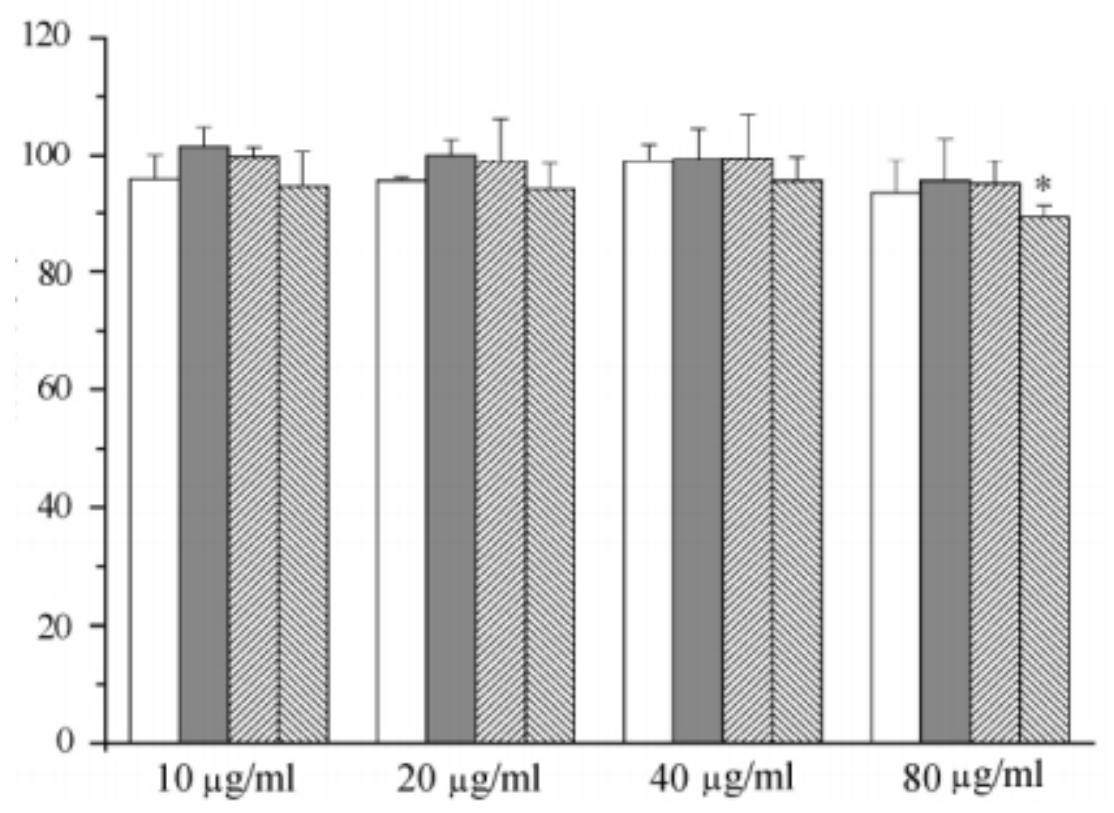

Figure 3. Viability analysis of different nanoparticle formulations at different concentrations.

In this study, CS and Man-CS coated FITCOVA-containing PLGA nanoparticles were prepared and their formulation properties were evaluated. The particle size of the nanoparticles is $(256 \pm 30) \sim(305 \pm 16) \mathrm{nm}$, and the drug loading is $(7.2 \pm 1.5) \% \sim 8$. $4 \pm 0.6) \%$. After the coating treatment, the size of PLGA nanoparticles increased, and the surface charge of the particles also changed from negative to positive, and was affected by the concentration of CS. The coating treatment reduced the burst effect of OVA, but also hindered the subsequent release process, indicating that its formulation and preparation process has yet to be optimized.

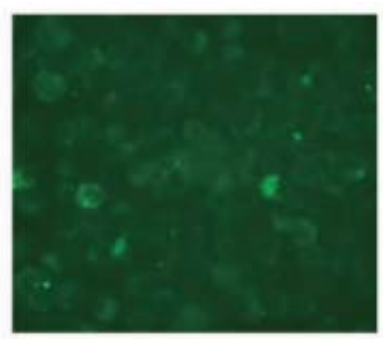

A

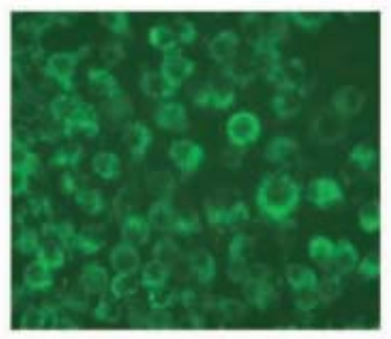

B

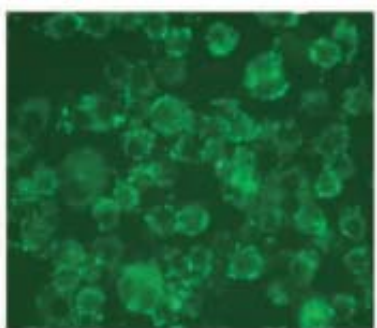

C

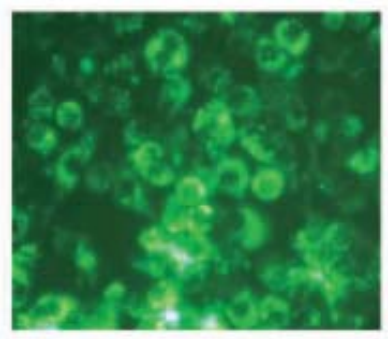

D

Figure 4. Cell uptake analysis by fluorescent microscopy 
MTT assays after co-incubation of macrophages with different nanocarriers showed that cell viability was above $90 \%$ with no significant cytotoxicity. The FITC-OVA uptake rate of macrophages from low to high was followed by FITC-OVA solution < FITC-OVAPLGA nanoparticles < CS-FITC-OVA-PLGA nanoparticles < Man-CSFITC-OVA-PLGA nanoparticles. The uptake of macrophages by nanoparticles is higher than that of solutions, which is related to the different mechanisms of uptake of liquids and nanoparticles [10]. In addition, since the surface of the cell membrane is generally negatively charged, the CS coating causes the surface of the FITC-OVA-PLGA nanoparticle to be positively charged, and then the expression of the receptorassociated mannose ligand on the surface of the macrophage significantly promotes cell uptake.

\section{Conclusion}

The mannose-modified cationic nanoparticle system carrying the model antigen was preliminarily established, which provided a basis for the study of anti-targeting cell targeting in vivo. Future efforts will focus on transferring this promising technology to the clinical settings

\section{References}

1. Mui, B. L.; Tam, Y. K.; Jayaraman, M.; Ansell, S. M.; Du, X.; Tam, Y. Y. C.; Lin, P. J.; Chen, S.; Narayanannair, J. K.; Rajeev, K. G., Influence of polyethylene glycol lipid desorption rates on pharmacokinetics and pharmacodynamics of siRNA lipid nanoparticles. Molecular Therapy-Nucleic Acids 2013, 2.

2. Seow, W. Y.; Liang, K.; Kurisawa, M.; Hauser, C. A., Oxidation as a facile strategy to reduce the surface charge and toxicity of polyethyleneimine gene carriers. Biomacromolecules 2013, 14 (7), 23402346.

3. Dong, Y.; Love, K. T.; Dorkin, J. R.; Sirirungruang, S.; Zhang, Y.; Chen, D.; Bogorad, R. L.; Yin, H.; Chen, Y.; Vegas, A. J., Lipopeptide nanoparticles for potent and selective siRNA delivery in rodents and nonhuman primates. Proceedings of the National Academy of Sciences 2014, 111 (11), 3955-3960.

4. Guerrero-Cázares, H.; Tzeng, S. Y.; Young, N. P.; Abutaleb, A. O.; Quiñones-Hinojosa, A.; Green, J. J., Biodegradable polymeric nanoparticles show high efficacy and specificity at DNA delivery to human glioblastoma in vitro and in vivo. ACS nano 2014, 8 (5), 5141-5153.

5. Ibraheem, D.; Elaissari, A.; Fessi, H., Gene therapy and DNA delivery systems. International journal of pharmaceutics 2014, 459 (1-2), 70-83.

6. Leonhardt, C.; Schwake, G.; Stögbauer, T. R.; Rappl, S.; Kuhr, J.-T.; Ligon, T. S.; Rädler, J. O., Single-cell mRNA transfection studies: delivery, kinetics and statistics by numbers. Nanomedicine: Nanotechnology, Biology and Medicine 2014, 10 (4), 679-688.

7. Laird, P. W.; Zijderveld, A.; Linders, K.; Rudnicki, M. A.; Jaenisch, R.; Berns, A., Simplified mammalian DNA isolation procedure. Nucleic acids research 1991, 19 (15), 4293.

8. Boussif, O.; Lezoualc'h, F.; Zanta, M. A.; Mergny, M. D.; Scherman, D.; Demeneix, B.; Behr, J.-P., A versatile vector for gene and oligonucleotide transfer into cells in culture and in vivo: polyethylenimine. Proceedings of the National Academy of Sciences 1995, 92 (16), 7297-7301. 
9. Kenworthy, A. K.; Hristova, K.; Needham, D.; Mclntosh, T. J., Range and magnitude of the steric pressure between bilayers containing phospholipids with covalently attached poly (ethylene glycol). Biophysical journal 1995, 68 (5), 1921-1936.

10. Krieg, A. M.; Yi, A.-K.; Matson, S.; Waldschmidt, T. J.; Bishop, G. A.; Teasdale, R.; Koretzky, G. A.; Klinman, D. M., CpG motifs in bacterial DNA trigger direct B-cell activation. Nature 1995, 374 (6522), 546. 11. Yu, Y.-C.; Roontga, V.; Daragan, V. A.; Mayo, K. H.; Tirrell, M.; Fields, G. B., Structure and dynamics of peptide- amphiphiles incorporating triple-helical proteinlike molecular architecture. Biochemistry 1999, 38 (5), 1659-1668.

12. Smith, J. D.; Cardwell, L. N.; Porciani, D.; Nguyen, J. A.; Zhang, R.; Gallazzi, F.; Tata, R. R.; Burke, D. H.; Daniels, M. A.; Ulery, B. D., Aptamer-displaying peptide amphiphile micelles as a cell-targeted delivery vehicle of peptide cargoes. Physical biology 2018, 15 (6), 065006.

13. Zhang, R.; Billingsley, M. M.; Mitchell, M. J., Biomaterials for vaccine-based cancer immunotherapy. Journal of Controlled Release 2018.

14. Zhang, R.; Kramer, J. S.; Smith, J. D.; Allen, B. N.; Leeper, C. N.; Li, X.; Morton, L. D.; Gallazzi, F.; Ulery, B. D., Vaccine Adjuvant Incorporation Strategy Dictates Peptide Amphiphile Micelle Immunostimulatory Capacity. The AAPS journal 2018, 20 (4), 73.

15. Zhang, R.; Leeper, C. N.; Wang, X.; White, T. A.; Ulery, B. D., Immunomodulatory vasoactive intestinal peptide amphiphile micelles. Biomaterials science 2018.

16. Zhang, R.; Leeper, C. N.; Wang, X.; White, T. A.; Ulery, B. D., Immunomodulatory vasoactive intestinal peptide amphiphile micelles. Biomaterials science 2018, 6 (7), 1717-1722.

17. Zhang, R.; Morton, L. D.; Smith, J. D.; Gallazzi, F.; White, T. A.; Ulery, B. D., Instructive Design of Triblock Peptide Amphiphiles for Structurally Complex Micelle Fabrication. ACS Biomaterials Science \& Engineering 2018.

18. Zhang, R.; Smith, J. D.; Allen, B. N.; Kramer, J. S.; Schauflinger, M.; Ulery, B. D., Peptide Amphiphile Micelle Vaccine Size and Charge Influence the Host Antibody Response. ACS Biomaterials Science \& Engineering 2018.

19. Zhang, R.; Smith, J. D.; Allen, B. N.; Kramer, J. S.; Schauflinger, M.; Ulery, B. D., Peptide Amphiphile Micelle Vaccine Size and Charge Influence the Host Antibody Response. ACS Biomaterials Science \& Engineering 2018, 4 (7), 2463-2472.

20. Zhang, R.; Ulery, B. D., Synthetic vaccine characterization and design. Journal of Bionanoscience 2018, $12(1), 1-11$.

21. Pack, D. W.; Hoffman, A. S.; Pun, S.; Stayton, P. S., Design and development of polymers for gene delivery. Nature reviews Drug discovery 2005, 4 (7), 581.

22. Torchilin, V. P., Recent advances with liposomes as pharmaceutical carriers. Nature reviews Drug discovery 2005, 4 (2), 145.

23. Minigo, G.; Scholzen, A.; Tang, C. K.; Hanley, J. C.; Kalkanidis, M.; Pietersz, G. A.; Apostolopoulos, V.; Plebanski, M., Poly-L-lysine-coated nanoparticles: a potent delivery system to enhance DNA vaccine efficacy. Vaccine 2007, 25 (7), 1316-1327.

24. Akinc, A.; Zumbuehl, A.; Goldberg, M.; Leshchiner, E. S.; Busini, V.; Hossain, N.; Bacallado, S. A.; Nguyen, D. N.; Fuller, J.; Alvarez, R., A combinatorial library of lipid-like materials for delivery of RNAi therapeutics. Nature biotechnology 2008, 26 (5), 561.

25. Bowie, A. G.; Unterholzner, L., Viral evasion and subversion of pattern-recognition receptor signalling. Nature Reviews Immunology 2008, 8 (12), 911.

26. Weide, B.; Carralot, J.-P.; Reese, A.; Scheel, B.; Eigentler, T. K.; Hoerr, I.; Rammensee, H.-G.; Garbe, C.; Pascolo, S., Results of the first phase I/II clinical vaccination trial with direct injection of mRNA. Journal of immunotherapy 2008, 31 (2), 180-188.

27. Weide, B.; Pascolo, S.; Scheel, B.; Derhovanessian, E.; Pflugfelder, A.; Eigentler, T. K.; Pawelec, G.; Hoerr, I.; Rammensee, H.-G.; Garbe, C., Direct injection of protamine-protected mRNA: results of a 
phase $1 / 2$ vaccination trial in metastatic melanoma patients. Journal of immunotherapy 2009, 32 (5), 498-507.

28. Xiang, S. D.; Selomulya, C.; Ho, J.; Apostolopoulos, V.; Plebanski, M., Delivery of DNA vaccines: an overview on the use of biodegradable polymeric and magnetic nanoparticles. Wiley Interdisciplinary Reviews: Nanomedicine and Nanobiotechnology 2010, 2 (3), 205-218.

29. Christensen, D.; Korsholm, K. S.; Andersen, P.; Agger, E. M., Cationic liposomes as vaccine adjuvants. Expert review of vaccines 2011, 10 (4), 513-521.

30. Kormann, M. S.; Hasenpusch, G.; Aneja, M. K.; Nica, G.; Flemmer, A. W.; Herber-Jonat, S.; Huppmann, M.; Mays, L. E.; Illenyi, M.; Schams, A., Expression of therapeutic proteins after delivery of chemically modified mRNA in mice. Nature biotechnology 2011, 29 (2), 154.

31. Siegwart, D. J.; Whitehead, K. A.; Nuhn, L.; Sahay, G.; Cheng, H.; Jiang, S.; Ma, M.; Lytton-Jean, A.; Vegas, A.; Fenton, P., Combinatorial synthesis of chemically diverse core-shell nanoparticles for intracellular delivery. Proceedings of the National Academy of Sciences 2011, 108 (32), 12996-13001.

32. Varkouhi, A. K.; Scholte, M.; Storm, G.; Haisma, H. J., Endosomal escape pathways for delivery of biologicals. Journal of Controlled Release 2011, 151 (3), 220-228. 